\title{
Lack of cellular and humoral immunological responses to oats in adults with coeliac disease
}

\author{
E K Janatuinen, T A Kemppainen, P H Pikkarainen, K H Holm, V-M Kosma,
} M I J Uusitupa, M Mäki, R J K Julkunen

Gastroenterological Unit, Department of Medicine, Kuopio University Hospital, Kuopio, Finland E K Janatuinen R J K Julkunen

Department of Clinical Nutrition, University of Kuopio, Kuopio, Finland

T A Kemppainen

M I J Uusitupa

Gastroenterological Unit, Department of Medicine, Tampere University Hospital, Tampere, Finland

P H Pikkarainen

Department of Paediatrics, Tampere University Hospital, Tampere, Finland K H Holm

Department of Pathology and Forensic Medicine, University of Kuopio, Kuopio, Finland V-M Kosma

Institute of Medical Technology, University of Tampere, Tampere, Finland

M Mäki

Correspondence to: Professor M Uusitupa, Department of Clinical Nutrition, University of Kuopio, PO Box 1627, SF-70211 Kuopio, Finland

Accepted for publication 5 October 1999

\begin{abstract}
Objective-Recent research suggests that oats do not harm intestinal villi in adults with coeliac disease. As the immunological effects of oats have not been examined in detail, it was decided to compare the immunological responses of a gluten free diet including oats with those of a conventional gluten free diet.

Design-A randomised controlled intervention study over 6-12 months.

Subjects-Forty adults with newly diagnosed coeliac disease and 52 with coeliac disease in remission were examined.

Intervention-The effects of a gluten free diet including oats and a conventional gluten free diet were compared.

Main outcome measures-Serum levels of gliadin and reticulin antibodies as well as numbers of intraepithelial lymphocytes (IELs) in intestinal mucosa were examined before and after the intervention.

Results-The rate of disappearance of gliadin and reticulin antibodies did not differ between the diet groups in patients with newly diagnosed coeliac disease. Oats also had no effect on gliadin or reticulin antibody levels in the patients with remission. The number of IELs decreased similarly regardless of the diet of newly diagnosed patients, and no increase in the number of IELs was found in the patients in remission with or without oats.

Conclusions-These results strengthen the view that adult patients with coeliac disease can consume moderate amounts of oats without adverse immunological effects.

(Gut 2000;46:327-331)
\end{abstract}

Keywords: coeliac disease; diet; oats; anti-gliadin antibodies; anti-reticulin antibodies; intraepithelial lymphocytes

Wheat, rye, and barley have harmful effects on the small intestinal mucosa of patients with coeliac disease, whereas maize and rice are harmless. ${ }^{12}$ It has been recommended that wheat, rye, barley, and also oats should be avoided. ${ }^{34}$ However, the place of oats in the coeliac diet has been debated. ${ }^{5-10}$ We did not find any detrimental consequences of oats on duodenal villous architecture, symptoms, or nutritional status in our previous 6-12 month study on the effects of oats in patients with coeliac disease in remission or with newly diagnosed disease. ${ }^{11}$ In a recent short term Irish study with a small number of patients in remis- sion and without any control group, oats showed no toxic or immunological effects either. ${ }^{12}$ Newly diagnosed patients were not included in that study.

The objectives of this study were to investigate in a controlled randomised study design ${ }^{11}$ whether oats in the diets of adult patients with newly diagnosed coeliac disease would have any effect on the rate of decrease in gliadin and reticulin antibody levels, as indicators of humoral immunology, or the number of intraepithelial lymphocytes (IELs), a marker of local immune response. The same variables were also measured in patients with coeliac disease in remission.

\section{Methods}

PATIENT POPULATIONS

The composition of the study population has been described in detail previously. ${ }^{11}$ The final study population consisted of two groups of patients: those with coeliac disease diagnosed earlier $(n=52)$ and newly diagnosed patients $(n=40)$.

The original diagnosis of coeliac disease in the previously diagnosed patients was based on the presence of subtotal or total villous atrophy of the duodenal mucosa before introduction of the gluten free diet. The inclusion criteria for these patients were age 18 or older and a normal or almost normal duodenal villous architecture after consumption of a gluten free diet for at least 12 months.

All newly diagnosed patients with subtotal or total villous atrophy without any reason for secondary villous atrophy diagnosed in the Kuopio University Hospital, Finland, were included in the study between 1 December 1988 and 31 December 1990. A patient was excluded if there was any medical condition that the investigator considered sufficiently serious to interfere with the conduction of the trial or to constitute an unacceptable risk to the patient.

Exclusion criteria for both groups were: previous or current corticosteroid treatment for the disease; history of complications of coeliac disease; any neurological, cardiovascular, pulmonary, metabolic, haematological, or endocrine disorder that could hinder participation in the trial; history of drug or alcohol abuse; mental impairment; lack of cooperation or refusal to take part in the trial. Patients were also excluded if the coeliac disease diagnosis

Abbreviations used in this paper: IEL, intraepithelial lymphocyte; EU/ml, enzyme immunosorbent assay unit. 
was not definite, for instance if there was any other reason for villous atrophy. Also patients whose diet included oats were excluded.

STUDY DESIGN AND DIETS

Patients were randomised by sex into either the oat consuming or the control groups. The clinical nutritionist gave both verbal and written instructions about the diet. Control patients received gluten free cereal free of charge (Raisio Factories-Melia Ltd, Raisio, Finland). The oat consuming groups (oat groups) received products supplemented with oats: two types of gluten free wheat starch flour including $50 \%$ oats, muesli including $60 \%$ oats, and rolled oat breakfast cereal. Some of the oat products used in this study were also commercially available. The goal for the daily intake of oats was 50-70 g. ${ }^{11}$ All oat products were made from three Finnish oat strains (Veli, Puhti, and Ryhti). The purity of the oats was regularly monitored. Analysis of gluten content was performed at the National Food Administration in Sweden (Statens Livsmedelsverk, Sweden). Gluten was analysed by a quantitative enzyme immunoassay using a specific monoclonal antibody to $\omega$-gliadin. This antibody detects all prolamins of wheat and rye but only part of barley prolamin. The prolamin of oats, avenin, is not detected by this antibody. All oat samples were gluten free.

\section{EXAMINATIONS}

Before the study, a screening gastroscopy was performed with an Olympus GIF Q20 end viewing gastroscope, and two duodenal biopsy specimens were obtained if possible using jumbo forceps (Olympus FB-13K). Specimens were fixed in $10 \%$ buffered formalin, processed by standard methods, and stained by van Gieson's method. ${ }^{13}$ The specimens were studied without knowledge of the patient's clinical status. IELs were identified and counted in specimens under a light microscope with a $\times 100 \mathrm{flat}$ field objective. Lymphocytes from the epithelium of the villi, or in cases of flat mucosa, from the surface epithelium were counted. In each specimen, nuclei were counted in at least 200 epithelial cells.

Serum for antibody testing was withdrawn at baseline and at one, three, six, and 12 months. IgA and IgG gliadin antibodies were measured using an inhouse solid phase enzyme linked immunosorbent assay (ELISA). ${ }^{14}$ The results were obtained from a standard curve established by dilutions of a positive reference serum and converted into concentrations of arbitrary ELISA units (EU/ml). Serum samples containing gliadin antibodies at higher concentrations than known healthy age matched controls, +2 $\mathrm{SD}$, were considered positive. Serum IgA R1-type reticulin antibodies were determined by an indirect immunofluorescence method, by using unfixed cryostat sections of rat kidney, liver, stomach, and heart as antigen. ${ }^{15}{ }^{16} \mathrm{~Pa}-$ tients' sera were screened at dilutions of 1:5 and 1:50 with fluorescein isothiocyanate conjugated antiserum to human $\operatorname{IgA}$ (goat antiserum; Sanoti Diagnostics Pasteur Inc, Chaska, Minnesota, USA; dilution 1:120). Positive sera were further titrated 1:50, 1:100, $1: 200,1: 500,1: 1000,1: 2000,1: 4000$, and $1: 8000$.

Food records, clinical assessments and laboratory screening were carried out concurrently. Endoscopies with duodenal biopsies were performed at baseline, six months, and 12 months for patients with newly diagnosed coeliac disease.

PATIENT WITHDRAWALS

There were 11 withdrawals: six in the remission group and five in the group of newly diagnosed patients. Two patients with dermatitis herpetiformis in the control and two in the oat group reported worsening of itching without any signs of dermatitis and therefore skin biopsies and histochemical analyses were not per-

Table 1 Effect of oats in the diet of adult patients with coeliac disease and gliadin and reticulin antibodies

\begin{tabular}{|c|c|c|c|c|c|c|}
\hline \multirow[b]{2}{*}{ Variable } & \multicolumn{3}{|c|}{ Coeliac disease in remission } & \multicolumn{3}{|c|}{ Newly diagnosed coeliac disease } \\
\hline & Oat group $(n=26)$ & Control group $(n=26)$ & $p$ Value & Oat group $(n=19)$ & Control group $(n=21)$ & $p$ Value \\
\hline \multicolumn{7}{|l|}{ Anti-gliadin IgA (EU/ml) } \\
\hline Baseline & $0.0(0-0.63)$ & $0.0(0-0.68)$ & & $0.55(0-10.0)$ & $0.62(0-10.0)$ & \\
\hline 1 month & $0.0(0-0.64)$ & $0.0(0-0.16)$ & & $0.27(0-10.0)$ & $0.29(0-4.31)$ & \\
\hline 3 months & $0.0(0-0.59)$ & $0.0(0-0.96)$ & & $0.14(0-1.8)$ & $0.0(0-7.46)$ & \\
\hline 6 months & $0.0(0-0.85)$ & $0.0(0-0.39)$ & & $0.0(0-1.9)$ & $0.0(0-1.13)$ & \\
\hline 12 months & - & - & & $0.0(0-1.8)$ & $0.0(0-1.45)$ & \\
\hline Change & $0.0(-0.47$ to 0.41$)$ & $0.0(-0.31$ to 0.03$)$ & 0.33 & $-0.73(-9.9$ to 0$)$ & $-0.57(-9.38$ to 0$)$ & 0.69 \\
\hline Group difference $(95 \% \mathrm{CI})$ & $0(0$ to 0.01$)$ & & & $0(-0.45$ to 0.53$)$ & & \\
\hline \multicolumn{7}{|l|}{ Anti-gliadin IgG $(\mathrm{EU} / \mathrm{ml})$} \\
\hline Baseline & $0.86(0-5.71)$ & $0.0(0-5.17)$ & & $7.82(0-30.8)$ & $3.49(0.65-61.2)$ & \\
\hline 1 month & $0.76(0-6.72)$ & $0.56(0-4.08)$ & & $6.13(0-26.5)$ & $5.41(0.58-69.8)$ & \\
\hline 3 months & $0.72(0-8.07)$ & $0.0(0-3.86)$ & & $3.95(0-11.3)$ & $2.76(0-22.1)$ & \\
\hline 6 months & $0.97(0-7.4)$ & $0.0(0-2.54)$ & & $2.03(0-6.23)$ & $1.97(0-9.28)$ & \\
\hline 12 months & - & - & & $1.15(0-6.97)$ & $1.04(0-9.2)$ & \\
\hline Change & $0.0(-1.21$ to 2.02$)$ & $0.0(-2.63$ to 0.86$)$ & 0.12 & -7.09 ( -29.85 to 0$)$ & $-2.99(-55.2$ to 0.53$)$ & 0.99 \\
\hline Group difference $(95 \% \mathrm{CI})$ & $0.18(0$ to 0.66$)$ & & & $1.28(-2.14$ to 4.04$)$ & & \\
\hline \multicolumn{7}{|l|}{ Anti-reticulin IgA (EU/ml) } \\
\hline Baseline & $0(0-50)$ & $0(0-50)$ & & $200(0-2000)$ & $200(0-4000)$ & \\
\hline 1 month & $0(0-5)$ & $0(0-50)$ & & $50(0-200)$ & $0(0-1000)$ & \\
\hline 3 months & $0(0-5)$ & $0(0-0)$ & & $0(0-200)$ & $0(0-200)$ & \\
\hline 6 months & $0(0-5)$ & $0(0-0)$ & & $0(0-0)$ & $0(0-8000)$ & \\
\hline 12 months & - & - & & $0(0-5)$ & $0(0-50)$ & \\
\hline Change & $0(-50$ to 0$)$ & $0(-50$ to 0$)$ & 1.0 & $-200(-2000$ to 0$)$ & $-175(-4000$ to 5$)$ & 0.79 \\
\hline Group difference $(95 \% \mathrm{CI})$ & $0(0$ to 0$)$ & & & $0(-50$ to 200$)$ & & \\
\hline
\end{tabular}

Values are median (range), except for differences in changes (as medians) between the groups; $95 \%$ confidence intervals (CI) are shown in parentheses; $\mathrm{p}$ values were calculated using the Mann-Whitney U test. 

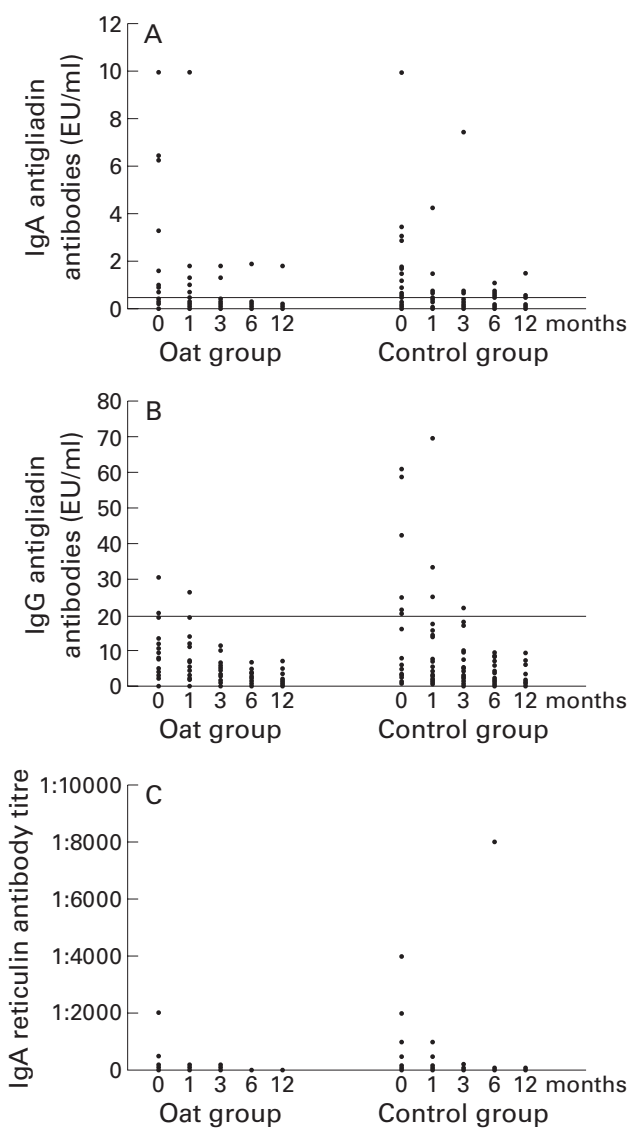

Figure 1 Titres of $\operatorname{Ig} A(A)$ and $\operatorname{Ig} G(B)$ gliadin antibodies and $\operatorname{Ig} A(C)$ reticulin antibodies in the oat and control groups of newly diagnosed coeliac patients determined at baseline, and at one, three, six, and 12 months after the institution of the diet. There were no significant differences between the groups ( $p>0.10$; Fisher test). Within group changes were significant for anti-gliadin $\operatorname{Ig} A(p=0.016$ and $p=0.002)$ and for reticulin antibodies $(p=0.001$ and $p=0.002)$ in both groups, and for anti-gliadin IgG in the control group $(p=0.031)$ (McNemar test). Normal levels of anti-gliadin Ig $A$ are $<0.5 \mathrm{EU} / \mathrm{ml}$ and of anti-gliadin IgG $<20 \mathrm{EU} / \mathrm{ml}$; normal values of $I g A$ reticulin antibody are titres $<5$. The horizontal line in $(A)$ and $(B)$ represent the upper cut off limit of normal values of IgA and IgG gliadin antibodies respectively. At 12 months, three patients (one in the oat consuming group and two in the control group) had positive $(>0.5)$ anti-gliadin IgA. All patients had normal anti-gliadin IgG levels. At 12 months, three patients (one in the oat consuming group and two in the control group) had anti-reticulin IgA levels of 5-50 EU/ml.

formed. Two patients in the oat group withdrew because of abdominal symptoms (one patient with earlier diagnosed disease and one newly diagnosed). They showed no clinical or histopathological evidence of deterioration of coeliac disease on examination of duodenal biopsy specimens. Three patients in the control group and two in the oat group refused to continue without giving any reason.

The study protocol was approved by the ethics committee of Kuopio University. All patients received written information about the trial, and verbal consent was obtained from each before they started the diet. Patients were also informed that they could withdraw from the study at any point.

\section{STATISTICAL ANALYSIS}

All the analyses were carried out with the intention to treat principle. Statistical analyses were performed using the SPSS-PC (SPSS Inc, Chicago, Illinois, USA) and CIA programs. ${ }^{17}$ Non-parametric tests were used when variables did not have a normal distribution and equal variance. The differences between the groups were assessed by the Mann-Whitney $U$ test. The results are presented as means (SD) or medians (range). The 95\% confidence intervals were calculated for the differences in change as median or as mean values between the two groups. The Fisher exact test was used to analyse the differences between the study groups in frequency and the proportion of categorised variables as normal values. The differences between the time points were analysed by the McNemar test.

\section{Results}

The mean oat intake was 43.6 (11.3) g/day at six months and 46.6 (13.3) g/day at 12 months for the newly diagnosed patients. The consumption of oats was 49.9 (14.7) g/day at six months for the patients in remission. Of the original study groups, $14(74 \%)$ newly diagnosed patients and $21(81 \%)$ patients in remission consumed more than $30 \mathrm{~g}$ of oats a day at the end of the study. ${ }^{11}$

\section{SERUM LEVELS OF GLIADIN AND RETICULIN ANTIBODIES}

At the beginning of the study, $17 \%$ of newly diagnosed patients in the oat group and $29 \%$ in the control group were anti-reticulin IgA negative, and $50 \%$ of them were anti-gliadin IgA negative in the oat group and $38 \%$ in the control group. Respectively $84 \%$ and $72 \%$ were anti-gliadin IgG negative. In the newly diagnosed patients the rates of disappearance of the antibodies were similar regardless of the diet (table 1, fig 1). The median levels of gliadin (IgA, $\operatorname{IgG})$ and reticulin ( $\operatorname{IgA})$ antibodies in the patients with coeliac disease in remission did not differ significantly at any point of the study in the oat group compared with the control group (table 1). The single high titre of

Table 2 Effect of oats in the diet of adult patients with coeliac disease on total intraepithelial lymphocytes (IELs)

\begin{tabular}{|c|c|c|c|c|c|c|}
\hline \multirow[b]{2}{*}{ Variable } & \multicolumn{3}{|c|}{ Coeliac disease in remission } & \multicolumn{3}{|c|}{ Newly diagnosed coeliac disease } \\
\hline & Oat group $(n=26)$ & Control group $(n=26)$ & p Value & Oat group $(n=19)$ & Control group $(n=21)$ & $p$ Value \\
\hline \multicolumn{7}{|l|}{ IEL count/100 epithelial cells } \\
\hline Baseline & $38.0(20)$ & $35.2(13.2)$ & & $58.2(19.2)$ & $60.3(18.6)$ & \\
\hline 6 months & $37.4(13.8)$ & $37.3(14.7)$ & & $34.3(15.6)$ & $36.0(14.5)$ & \\
\hline 12 months & - & - & & $34.5(12.9)$ & $37.9(22.4)$ & \\
\hline Change & $0.6(21.8)$ & $2.0(11.7)$ & 0.94 & $-23.8(23.3)$ & $-21.7(14.5)$ & 0.84 \\
\hline Group difference $(95 \% \mathrm{CI})$ & $-2.6(12.3$ to 7.2$)$ & & & $-2.1(-14.4$ to 10.2$)$ & & \\
\hline
\end{tabular}

Values are means (SD); 95\% confidence intervals (CI) are shown in parentheses; $p$ values were calculated using the Mann-Whitney U test. 
anti-reticulin $\operatorname{IgA}$ in one patient of the control group at six months (fig 1C) can be explained by temporary non-compliance with the diet.

\section{INTRAEPITHELIAL LYMPHOCYTES}

In the patients with newly diagnosed coeliac disease, the IEL counts in the oat group and the control group decreased at six and 12 months at a similar rate (table 2). There were no significant differences between the study groups at any point of the study.

The mean IEL counts of the patients in remission in the oat and control groups stayed at the same level for six months (table 2).

\section{Discussion}

We have previously reported the suitability of oats for adult coeliac patients as a part of their otherwise gluten free diet. ${ }^{11}$ This was based on findings of no harmful effects on duodenal mucosa, laboratory variables, nutritional status, and subjective wellbeing. Our present results show that oats do not prevent normalisation of gliadin and reticulin antibodies or the number of IELs in the duodenal mucosa of newly diagnosed patients. The use of oats in the diet also produces no unfavourable local or humoral immunological effects in adults with coeliac disease in remission. In a recent study of 10 patients in remission, $50 \mathrm{~g}$ of oats a day for three months did not cause any significant changes in the number of IELs or the levels of gliadin antibodies. ${ }^{12}$ The same was also true in two recent studies on patients with dermatitis herpetiformis treated with a diet including oats. ${ }^{18} 19$ The results of the present report and the former studies ${ }^{11}{ }^{12}$ extend our knowledge of the effects of oats in coeliac disease and emphasise its safety as a part of a gluten free diet for adult patients with coeliac disease.

In coeliac disease, patients are sensitised to gluten, and serum gliadin antibodies are often detected. In children, gliadin antibodies are highly sensitive and specific for diagnosis of coeliac disease ${ }^{19}{ }^{20}$ In the elderly, positive values for gliadin antibodies do not always indicate undetected coeliac disease, but may represent a normal response to dietary antigens. ${ }^{21}$ On the other hand, serum reticulin antibodies are both sensitive and disease specific. ${ }^{22}$ In our hands, classical serum reticulin antibody titres correlate well with endomysial antibodies. ${ }^{1622}$

Serum IgA class gliadin antibody concentrations have been used as evidence of dietary compliance as well as as markers of mucosal damage in patients with coeliac disease who are eating gluten. ${ }^{23}{ }^{24}$ In adults with coeliac disease, after two years on a strict gluten free diet, no significant difference in serum IgA class gliadin antibody concentrations has been found compared with normal subjects. ${ }^{25}$ Positive values for both IgA and IgG class gliadin antibodies seem to be more related to the lack of improvement in the jejunal lesions than to the strictness or duration of gluten withdrawal. ${ }^{26}$

Serum levels of reticulin antibodies have been shown to be reliable for predicting mucosal relapse in coeliac disease. In a study of postpubertal patients with coeliac disease, a diet containing normal amounts of gluten caused both histological relapse of coeliac disease and an increase in reticulin antibody levels in all patients, whereas gliadin antibody was positive only in $89 \%$ of these cases. ${ }^{27}$

High IEL counts in the jejunal mucosa of untreated patients with coeliac disease are typical, but some patients with coeliac disease in remission may still have high numbers of IEL. ${ }^{28}{ }^{29}$ There is a dose dependent effect of ingestion of gliadin on jejunal villous morphometry and the numbers of IELs in children with coeliac disease..$^{30}$ In the present study, the IEL count was similar at the end of the follow up in all diet groups, confirming the safety of oats in patients with coeliac disease.

In vitro culture experiments have shown avenin, the prolamin of oats, to activate intestinal cell mediated immunity in coeliac disease. ${ }^{31}{ }^{32}$ Large quantities of avenin have also induced lymphocyte infiltration when instilled in the rectal mucosa of coeliac patients. ${ }^{32}$ These observations are not in accordance with clinical studies, which have shown reasonable amounts of oats - that is, avenin - to be well tolerated and not to cause mucosal damage of the small intestine $e^{112181933}$ or immune activation in patients with either coeliac disease or dermatitis herpetiformis in remission. ${ }^{12} 181933$

It has been suggested that conventional oats may be contaminated with wheat during growth or the milling process. ${ }^{18}$ The purity of the oat products used in previous studies $^{11} 12181933$ and the present one was confirmed by chemical analysis, which adds reliability to the favourable results of tolerance to oats both in coeliac disease and dermatitis herpetiformis. However, it is possible to ingest small amounts of gluten through eating contaminated processed food based on any initially gluten free products. In addition, subconscious and conscious lapses in diet have also been considered in interpreting high antibody titres during dietary treatment. ${ }^{34}$ The few withdrawals in our study do not seem to have been caused by oats, as the products were found to be free of contamination in the chemical analyses.

In this study, patients with severe coeliac disease - that is, those who needed corticosteroids to obtain remission-were excluded. In the Irish study, one patient who was very sensitive to trace amounts of gluten also tolerated oats. ${ }^{12}$ Two other patients showed evidence of relapse during a separate gluten "microchallenge", although oats did not have any harmful effects. ${ }^{12}$ Thus even patients who are exceptionally sensitive to gluten seem to be able to consume oats. However, no information is available on oat tolerance in cases of refractory coeliac disease. More clinical studies are needed to ensure the safety of oats when consumed permanently in a coeliac diet as well as to determine the effect of larger amounts of oats.

In conclusion, our results show that oats used as part of an otherwise gluten free diet in adults with coeliac disease in remission do not cause any changes in humoral or local immunological responses, as measured by levels of gliadin and reticulin antibodies or 
numbers of IELs. More importantly, our report shows that a gluten free diet containing oats normalises gliadin and reticulin antibodies as well as the number of IELs in the intestinal mucosa of newly diagnosed patients at the same rate as in patients consuming a conventional strict gluten free diet. These results further support the conception that adult coeliac patients can eat moderate amounts of oats without harmful effect.

The work was supported by grants from the Yrjö Jahnsson Foundation and the Finnish Gastroenterological Association. The oats products were supplied by Raisio Factories-Melia Ltd, Raisio, Finland and given to the study patients free of charge.

1 Dicke WK. Coeliakie. Een onderzoek nar de nadelige invloed van sommige graansoorten op de lijder aan coeliakie. Thesis, Utrecht 1950.

2 Dicke WK, Weijers HA, van de Kamer JH. Coeliac disease. The presence in wheat of a factor having a deleterious effect in cases of coeliac disease. Acta Paediatr 1953;42:3543.

3 Van de Kamer JH, Weijers HA, Dicke WK. Coeliac disease: an investigation into the injurious constituents of wheat in an investigation into the injurious constituents of wheat in connection with their action on pa
disease. Acta Paediatr 1953;42:223-31.

4 Kendall MJ, Cox PS, Schneider R, et al. Gluten subfractions in coeliac disease. Lancet 1972;ii:1065-7.

5 Moulton ALC. The place of oats in the coeliac diet. Arch Dis Child 1958;34:51-5.

6 Anderson CM, Gracey M, Burke V. Coeliac disease. Some still controversial aspects. Arch Dis Child 1972;47:292-8.

7 Dissanayake AS, Truelove SC, Whitehead R. Lack of harmful effect of oats on small-intestinal mucosa in coeliac disease. $B M F$ F 1974;4:189-91.

8 Baker PG, Read AE. Oats and barley toxicity in coeliac patients. Postgrad Med f 1976;52:264-8.

9 Baker PG. Oats and coeliac disease. BMF 1974;4:588-9.

10 Kumar PJ, O'Donoghue DP, Stenton K, et al. Reintroduction of gluten in adults and children with treated coeliac disease. Gut 1979;20:743-9.

11 Janatuinen EK, Pikkarainen PH, Kemppainen TA, et al. A comparison of diets with and without oats in adults with comparison of diets with and without oats in

12 Srinivasan U, Leonard N, Jones E, et al. Absence of oats toxicity in adult coeliac disease. BMf 1996;313:1300-1.

13 Bancroft JD, Stevens A, eds. Theory and practice of histological techniques. 3rd ed. Edinburgh, London, Melbourne, New York: Churchill Livingstone, 1982:112-13.

14 Savilahti E, Viander M, Perkkiö M, et al. IgA antigliadin antibodies: a marker of mucosal damagein childhood coeliac disease. Lancet 1983;i:320-2.

15 Mäki M, Hällstrom O, Vesikari T, et al. Evaluation of serum IgA-class reticulin antibody test for detection of childhood coeliac disease. F Pediatr 1984;105:901.

16 Sulkanen S, Halttunen T, Marttinen A, et al. Autoantibodies in coeliac disease: importance of fibroblasts. $\mathcal{F}$ Pediatr Gastroenterol Nutr 1998:27:206-13.
17 Altman DG, Gardner MJ. Calculating confidence intervals for means and their differences. In: Gardner MJ, Altman DG, ed. Statistics with confidence - confidence intervals and statistical guidelines. Belfast: The Universities Press Ltd, 1989:20-7.

18 Hardman CM, Garioch JJ, Leonard JN, et al. Absence of toxicity of oats in patients with dermatitis herpetiformis. $N$ Engl Med f 1997;337:1884-7.

19 Reunala T, Collin P, Holm K, et al. Tolerance to oats in dermatitis herpetiformis. Gut 1998:43:490-3.

20 McMillan SA, Haughton DJ, Biggart JD, et al. Predictive value for coeliac disease of antibodies to gliadin, endomysium, and jejunum in patients attending for jejunal biopsy. sium, and jejunum in patie

21 Uibo O, Uibo R, Kleimola V, et al. Serum IgA anti-gliadin antibodies in an adult population sample. High prevalence without coeliac disease. Dig Dis Sci 1993;38:2034-7.

22 Maki M. The humoral immune system in coeliac disease. Baillieres Clin Gastroenterol 1995;9:231-49.

23 Hällstrom O. Comparison of IgA-class reticulin and endomysium antibodies in coeliac disease and dermatitis herpetiformis. Gut 1989;30:1225-32.

24 Kelly CP, Feighery CF, Gallagher RB, et al. Mucosal and systemic IgA anti-gliadin antibody in coeliac disease. Contrasting patterns of response in serum, saliva, and intestinal secretions. Dig Dis Sci 1991;36:743-51.

25 Hill PG, Thompson SP, Holmes GK. IgA anti-gliadin antibodies in adult coeliac disease. Clin Chem 1991;37:647-50.

26 Volta U, Corazza GR, Frisoni M, et al. IgA antigliadin antibodies and persistence of jejunal lesions in adult coeliac disease. Digestion 1990;47:111-14

27 Mäki M, Lahdeaho ML, Hällstrom O, et al. Postpubertal gluten challenge in coeliac disease. Arch Dis Child 1989;64: 1604-7.

28 Ferguson A, Murray D. Quantitation of intraepithelial lymphocytes in human jejunum. Gut 1971:2:988-94.

29 Holm HH. Correlation of HLA-DR alleles to jejunal mucosal morphology in healthy first-degree relatives of coeliac patients. Eur 7 Gastroenterol Hepatol 1993;5:35-9.

30 Catassi C, Rossini M, Ratsch IM, et al. Dose dependent effects of protracted ingestion of small amounts of gliadin in coeliac disease children: a clinical and jejunal morphometric study. Gut 1993:34:1515-19.

31 Leone NA, Mazzarella G, Giacci C, et al. Oats prolamines in vitro activate intestinal cell-mediated immunity in coeliac disease. In: Collin P, Mäki M, eds. All on coeliac disease. Seventh International Symposium on coeliac disease. Tampere: Lege Artis, 1996:69.

32 Troncone R, Mazzarella G, Leone A, et al. Oats prolamines are immunogenic for the coeliac intestinal mucosa. Proceedings of the 12th meeting, working group on prolamin analysis and toxicity. Tubingen: University of Tubingen, 1997:73-82.

33 Feighery C, Srinivasan U, Carolan J, et al. Oats challenge in vivo does not activate coeliac disease as determined by mmunohistological markers. In: Lohiniemi S, Collin P, Mäki M, eds. Changing features of coeliac disease. Tampere: The Finnish Coeliac Society, 1998:103-8.

34 Ciacci C, Mazzacca G. Unintentional gluten ingestion in coeliac patients. Gastroenterology 1988:115:243-50.

35 Greco L, Mayer M, Ciaccarelli G, et al. Compliance to a gluten-free diet in adolescent, or "What do 300 coeliac adolescents eat every day?". Int f Gastroenterol Hepatol 1997:29:305-11. 